SUMMARY

$x$. The dietary department should be a separate unit with its own personnel ; the head of the department should be a trained dietitian.

2. Sufficient money should be allowed in the hospital budget for the purchase of adequate amounts of good-quality foods.

3. Menus should be so arranged that suitable foods of equivalent value are available for a variety of conditions.

4. Efficient personnel must be encouraged to enter the hospital service. Good facilities and equipment are necessary for them to carry out their duties satisfactorily.

5. The therapeutic diet kitchen should be completely separate from the main kitchen and nurses should be given the opportunity to gain experience in this branch of medical work.

6. A small metabolic ward is necessary if accurate work is to be carried out.

\title{
Organization of a Diet Department. Out-Patients
}

\author{
By J. H. Croom, Assistant Physician, Royal Infirmary, \\ Edinburgh
}

On 31 December 1947, 10,234 persons in Scotland were drawing the extra rations allowed to the diabetic (Croom, 1950). By September 195r, this number had increased to 12,038 . It has been estimated, following the mass survey undertaken in 1947, by the United States Public Health Service at Oxford, Massachussets, that, for every diabetic who is aware of his condition, there is another who is not (Wilkerson \& Krall, 1947). If one believes that some dietary restriction is desirable in the diabetic, these figures alone suggest that the careful organization of a dietetic out-patient department is worth while. In the Dietetic Out-Patient Department, Royal Infirmary, Edinburgh, during $195 \mathrm{I}$, an average of thirtyone new diabetic patients were seen each month while over a thousand return visits were paid each month by patients suffering from this condition.

The life insurance companies have clear statistical proof that the expectation of life in the obese is below average and any practising clinician is aware of the numerous complications of the obese state, hypertension, recurrent bronchitis, digestive disturbances, impaired carbohydrate tolerance, varicose veins and many orthopaedic complaints. In addition, it is probably fair to say that no surgeon would willingly perform an operation, not immediately essential, on an obese patient before preliminary treatment of the obesity. If, then, an out-patient department dealt only with cases of diabetes mellitus and obesity it would make an important contribution to public health. Though these two conditions form a substantial proportion of the routine work of such a department, the staff have to advise dyspeptics, sufferers from various disorders of the bowels, and those 
requiring to restrict their sodium intake or to increase the protein content of their diet. Time is required to teach patients to consume a properly balanced diet, and to investigate the food habits of those in whom a deficiency is suspected.

The diabetic or obese patient benefits considerably by regular attendance at an organized clinic where he gets to know the staff and to feel that a personal interest is taken in his progress. Diabetics should attend for life, obese patients certainly for some months. In Edinburgh we do not encourage dyspeptics to pay more than two visits, one to receive dietetic instruction and another, perhaps a month later, to ensure that they have understood what they have been taught and have no further queries. Most other patients require one visit only.

It is obviously desirable that all hospitals and clinics should be attractive. This is doubly important in an out-patient department, where many patients are going to be invited to attend at regular intervals, perhaps for life.

Careful planning and organization can do much to ensure that the service provided is acceptable to the patient. The busy housewife or wage earner must feel that time is not being wasted. The lonely old lady, living alone, perhaps a little deaf and slightly bewildered, must feel that the staff are genuinely anxious to see her and are not merely trying to get rid of her in the shortest possible time.

The Dietetic Out-Patient Department at the Royal Infirmary, Edinburgh, has been in existence for over 25 years. It is associated with the School of Dietetics and is in the charge of the Professor of Therapeutics. This clinic is large, with records of approximately 44,500 patients and an average weekly attendance of 400 . It is open daily and receives patients not only from the staff of the hospital but also from general practitioners who send patients directly to the clinic. There has always been a relatively large dietetic staff of three trained dietitians under the supervision of a superintendent dietitian. There are, in addition, three dietetics students who receive instruction for 3 months at a time during their practical training.

A physician attends daily for approximately $2 \mathrm{~h}$ in the morning. He sees patients who the dietitians think require his attention and all new cases sent directly from outside practitioners. This organization is held to give better service than the system where all cases are seen by doctors on each occasion, and where only those who require some change in their diet are referred to the dietitian. Under our system more time is available and patients are able to discuss minor variations in their menu quietly and sympathetically with people they get to know and who are able to give expert advice to suit each individual's requirements.

Once a week a clinic is held in the evening from 6 to 8 p.m. primarily for people who are working all day. This is extremely popular, particularly with men suffering from diabetes.

On three afternoons a week one of the dietitians visits patients in their homes. This is of great value and is greatly appreciated by patients and their relatives. All new patients suffering from diabetes are visited within a month of their first attendance. This enables a more accurate assessment of their economic status to be made. More detailed instruction can be given to the housekeeper who actually 
prepares the food, and alterations in the diet may be suggested to suit cooking facilities and the patient's hours of work. Elderly and feeble patients, to whom the journey to the clinic is an ordeal, are visited about every 3 months. An attempt is made to visit the homes of defaulting patients who have not replied to the post-cards sent as a routine to those who have failed to report. Seldom are these visits resented, and most patients are gratified to find that such an active interest is taken in their well-being.

The Edinburgh Royal Infirmary serves a large area in Fife, the Lothians and the Scottish Borders as well as Edinburgh and District. It had been felt for long that clinics at the periphery 'would save patients' time and money and possibly reduce the numbers attending the clinic at the Royal Infirmary. Lawrence (195r) put forward a scheme for regional centres to treat diabetes. Under this scheme out-patient departments are to be situated throughout the area at the regional general hospitals and staffed by interested local consultants, dietitians and nurses. This has the great advantage that these patients are treated by the same people both as in-patients and as out-patients.

In the South-Eastern Region (Scotland) a different method has been adopted. A physician and dietitian from the Royal Infirmary travel each month to Kirkcaldy, Dunfermline, Haddington and Galashiels. I have been fortunate enough to be in charge of these peripheral clinics since their inception in January 1949. They are regarded as part of the service provided by the Edinburgh Royal Infirmary, and patients are transferred from one to the other as the need arises.

The clinics are accommodated at local hospitals in the closest liaison with local consultants and practitioners. Should a case require admission to the local hospital, the consultants have access to the records of the out-patient department. When a patient, who requires further observation, leaves the local hospital he is referred to the clinic for supervision. These clinics are situated in centres, on main bus routes, convenient for the country districts which they serve.

Help is obtained from the hospital car service organized by the Women's Voluntary Service in conjunction with the British Red Cross Society. This service is a great boon to the elderly and infirm. Those responsible for its organization have been most co-operative and efficient.

The accommodation required in the local hospital consists of two consulting rooms, a waiting room, a room for weighing patients and for testing urine, and a room for a secretary. In two of these clinics the secretary has to sit in one of the consulting rooms owing to lack of other suitable accommodation. This is not a satisfactory arrangement as it destroys the sense of privacy which the patient should feel.

Small supplies of insulin, scales and syringes are held at each clinic for issue to new diabetic patients. Supplies of stationery are maintained at each centre.

The staff, apart from the dietitian and physician, are local residents. The local hospital provides the services of a sister, and, in most instances, of a junior nurse to assist with weighing and with urine testing. The local Board of Management supplies a secretary. It leads to greater efficiency if the staff develop a simple 
routine in the reception of patients. It is wise to begin with a very small number of patients. Once the staff is familiar with the routine much larger numbers can be accommodated with greater efficiency.

An appointments system is not used at the large out-patient department of the Edinburgh Royal Infirmary. It is felt that patients should know that the department is open daily and that they are free to come at any time if they want help. This department is open daily so that patients can be seen as frequently as required. This unquestionably avoids admission to the infirmary in many cases and thus relieves the pressure on hospital beds. At the peripheral clinics, which are open only once a month, patients are seen by appointment. Every effort is made to consider the patient's convenience. The bus time-table and hours of work are taken into consideration. At one centre, where there is a good hospital car service, appointments are made in car-loads.

Records, both at the central and peripheral clinics are kept in typescript with the exception of the original history taken by a dietitian or by a dietetics student on the patient's first attendance. All subsequent notes are typed. The provision of adequate secretarial help is essential for the running of any out-patient department of this sort. A register is kept of diseases. It is thus easy to analyse statistically the incidence of the various diseases seen at the clinic.

\section{SUMMARY}

In planning a dietetic out-patient department the following points should be borne in mind :

I. The primary function is to provide advice on dietetics. Therefore the staff of dietitians should be as large as possible.

2. Since many of the patients will have to attend regularly, it is most important that the accommodation be attractive and that the staff make the patients feel at home and have a genuine interest in them as individuals.

3. A patient attending regularly for one condition may develop some intercurrent disease or degenerative process. The dietitians and physician must be aware of this and be prepared to take any necessary action.

4. The average person will co-operate better if he feels that a genuine interest is being taken in his welfare, for example by home visiting, provision of transport to the clinic, or provision of a clinic conveniently near his home.

5. Efficient secretarial help is essential.

6. Time spent in developing a fixed routine for the reception of patients is not wasted. When it has been developed more time is available for the personal interview by the dietitian or physician.

7. When planning an out-patient dietetic department it is wise to assume that the number of patients will increase markedly in the future. Not only is the number of diabetic patients increasing, but more interest is now also being taken in the dietetic treatment of obesity, of hypertension and of congestive cardiac failure and in the education of the general population in sound food habits. 
My thanks are due to Professor D. M. Dunlop and Miss A. Buchan, who have taught and encouraged me in my work in the Dietetic Department of the Edinburgh Royal Infirmary, and to Miss A. Buchan and Dr A. P. Meiklejohn for advice in the preparation of this paper.

REFERENCES

Croom, J. H. (1950). Edinburgh med. $\not$. 57, 185 .

Lawrence, R. D. (195I). Lancet, 260, 1318.

Wilkerson, H. L. C. \& Krall, L. P. (1947). F. Amer. med. Ass. 135, 209.

\section{Instruction of the Patient Attending the Dietetic Department of a Hospital}

\section{By Ella ScotT, Senior Dietitian, The London Hospital, Whitechapel}

When a patient has to assume responsibility for his own diet, either as an outpatient or on discharge from hospital, the information given to him must be practical and easily understood. The dietitian must consider carefully the factors that will influence and help the patient to carry out the instructions. These factors apply both to a normal diet and to a therapeutic diet, which is, after all, only a modification of the normal.

\section{Factors to be understood before instructing the patient}

Income is most important, it controls the standard of living to a great extent and must therefore be given full consideration in relation to the diet. Unfortunately, food is usually the first essential to suffer restriction when the income is low or reduced. The nationality, racial habits and religious principles of the patient affect both the choice and preparation of the food and play a large part in deciding the eating habits of the individual. Home conditions influence the management of the diet. Space for storage of food, larder accommodation, cooking facilities and water supply all determine the selection and preparation of the food. The occupation and daily routine affect both calorie intake and the planning of meals. Extra rations of certain foods are allowed for men doing heavy manual labour, the agricultural worker is allowed an extra allowance of cheese. The hours of work may be in shifts. For the patient on a special diet this may mean detailed adjustments each week. Canteen facilities, time allowed for meals, and the distance between the home and the place of work may also determine the type of meal.

The dietitian must have a thorough knowledge of the current rations and of the available unrationed foods and of their cost. This is necessary when giving advice on either the normal or the therapeutic diet. In the latter instance it is essential to have a thorough knowledge of the regulations of the Ministry of Food governing the issue of special rations to those requiring therapeutic diets. Instructions contrary to the views and habits of the patients are seldom obeyed. 